\title{
Atypical Olfactory Neuroblastoma Presenting as Sinonasal Polyposis with Nasopharyngeal Extension: A Case Report
}

\author{
Jagdish Prasad Purohit, Chandra Bhan*, Bhoopendra Singh, Ajay Pratap Singh, \\ Siva Selvaraj, Manish Pandey, Vijay Krishna Sharma \\ Department of ENT, Head and Neck Surgery, MLB Medical College, Jhansi, India \\ Email: ${ }^{*}$ chandrabhan.prajapati@gmail.com, bhoopimedico@gmail.com, chandrabhanprajapati1@gmail.com, \\ dr.aps27@gmail.com, drsivaselvaraj@gmail.com, selvarajk.siva@gmail.com, \\ chandrabhan.prajapati1@gmail.com
}

Received 20 July 2015; accepted 27 September 2015; published 30 September 2015

Copyright (C) 2015 by authors and Scientific Research Publishing Inc.

This work is licensed under the Creative Commons Attribution International License (CC BY). http://creativecommons.org/licenses/by/4.0/

(c) (i) Open Access

\begin{abstract}
Background: Olfactory neuroblastoma also known as Esthesioneuroblastoma (ENB) is a tumor arising from the basal layer of olfactory epithelium in the superior recess of the nasal cavity in the region of cribriform plate. Incidence peaks once in 11 - 20 years of age and again in 50 - 60 years of age. It is equally found in men and women. Aim: The main aim of this case report is to characterize the clinical features of ENB showing nasopharyngeal involvement and its importance in the differential diagnosis of sinonasal neuroendocrine malignancies. Case presentation: Two cases are reported. The first case was a 21-year-old male with symptoms of nasal obstruction, recurrent epistaxis, nasal discharge, headache and paresthesia over the face with an evolution of 1 year with no previous history of trauma. Diagnostic nasal endoscopy revealed reddish gray mass filling bilateral nasal cavities. Posterior rhinoscopy revealed mass coming through choanae and filling the nasopharynx. Endoscopic resection of tumor was done and postoperatively all the symptoms of the patient resolved. The second case was a 62-year-old male with complaints of nasal obstruction, nasal discharge, recurrent epistaxis, anosmia and difficulty in swallowing. On computed tomography, anteriorly, the mass was extending up to external nares and posteriorly up to the nasopharynx, superiorly up to nasal roof and inferiorly up to hard palate. No obvious erosion of floor of anterior cranial fossa was seen. No intracranial extension was seen. Surgery was advised but patient refused to undergo any treatment. Conclusion: This study highlights the characteristics and clinical features of ENB showing nasopharyngeal involvement and their importance in the differential diagnosis of sinonasal neuroendocrine malignancies.
\end{abstract}

${ }^{*}$ Corresponding author.

How to cite this paper: Purohit, J.P., Bhan, C., Singh, B., Singh, A.P., Selvaraj, S., Pandey, M. and Sharma, V.K. (2015) Atypical Olfactory Neuroblastoma Presenting as Sinonasal Polyposis with Nasopharyngeal Extension: A Case Report. International Journal of Otolaryngology and Head \& Neck Surgery, 4, 394-400. http://dx.doi.org/10.4236/ijohns.2015.45064 


\section{Keywords}

\section{Esthesioneuroblastoma, Cribriform Plate, Neuroectodermal}

\section{Introduction}

Olfactory neuroblastoma is an uncommon tumor of neuroectodermal origin, arising from basal cells of the olfactory neuroepithelium [1] [2]. Olfactory neuroblastoma represents less than 5 percent of all sinonasal malignancies. It is histologically similar to adrenal or sympathetic ganglionic neuroblastomas and retinoblastomas. The incidence of this tumor has a bimodal distribution with peaks at 20 and 50 years of age [3]. Unlike most of the sinonasal malignancies, it is equally distributed in women and men. The non-specific symptoms of nasal obstruction, recurrent epistaxis, hyposmia, headache and the special anatomical location of the tumor often lead to a diagnosis of benign paranasal disease thus delaying the correct diagnosis [4]. As the tumor grows, it tends to spread submucosally in all directions to involve the paranasal sinuses, nasal cavity, oral cavity, the orbits, and the brain [5] [6]. Tumor cells are mitotically active and developing into sustentacular and neuronal cells. There exist variable neurofibrillary materials. Neuro endocrine tumor is capable of causing paraneoplastic syndromes by secreting peptides.

\section{Objective}

This study highlights the characteristics and clinical features of ENB showing nasopharyngeal involvement and their importance in the differential diagnosis of sinonasal neuroendocrine malignancies.

\section{Case Report}

\subsection{Case 1}

A 21years old male came to our hospital complaining of nasal obstruction, recurrent epistaxis, nasal discharge, headache and paresthesia over the face with an evolution of 1 year with no previous history of trauma. Diagnostic nasal endoscopy revealed reddish gray mass filling the posterior part of the bilateral nasal cavities. Posterior rhinoscopy revealed mass coming through choanae and filling the nasopharynx. In the medical history, the patient had no reports of viral infections in childhood or other systemic illness. No family history of any hereditary diseases. Patient also denied any alcohol consumption or tobacco use. Cervical lymph node enlargement was not found on palpation.

Conventional X-rays and computed tomography (CT) revealed nasal cavities, bilateral maxillary, frontal, ethmoidal and sphenoidal sinuses soft tissue opacity. Lesion was extending in the posterior nasal cavity, upper part of posterior nasal septum and nasopharynx. Severe deossification of intervening bones was found. Both osteomeatal units were blocked and lamina papyracea were intact. Crista galli was normal (Figure 1 and Figure 2).

Microscopic examination of the incisional biopsy and surgical specimen showed cellular tissue consisting of uniformly small cells with round dense nuclei and scanty eosinophilic cytoplasm. Cells were arranged to form compact masses with occasional rosettes and fibrillary reticular background, separated by loose fibro-vascular stroma. Incisional biopsy suggested diagnosis towards olfactory neuroblastoma (Figure 3).

Patient was thoroughly evaluated; endoscopic removal of mass from the nasal cavity, maxillary, ethmoidal, sphenoidal and frontal sinuses and nasopharynx was done. Posterior part of nasal septum was removed. No adjuvant treatment was given. Patient became asymptomatic after surgery and during follow up.

\subsection{Case 2}

A 62 years old male patient came to our hospital with complaints of nasal obstruction, nasal discharge, recurrent epistaxis, anosmia and difficulty in swallowing for 10 years. Anterior rhinoscopy revealed reddish gray mass filling bilateral nasal cavities. Posterior rhinoscopy revealed mass coming through choanae and filling the nasopharynx. Mass was pushing hard and soft palate downwards compromising the oral cavity space (Figure 4). In the medical history of the patient there were no reports of trauma or other systemic illnesses. No family history of hereditary diseases was there. He gave history of alcohol consumption and smoking. Cervical lymph node enlargement was not detected on palpation. 


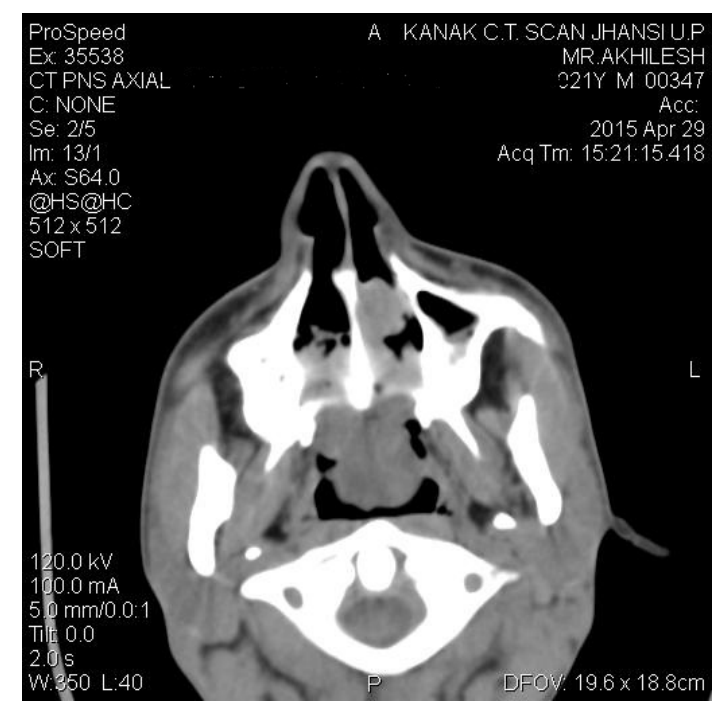

Figure 1. Case 1: CT PNS axial view showing tumor occupying the nasal cavity and extending up to nasopharynx.

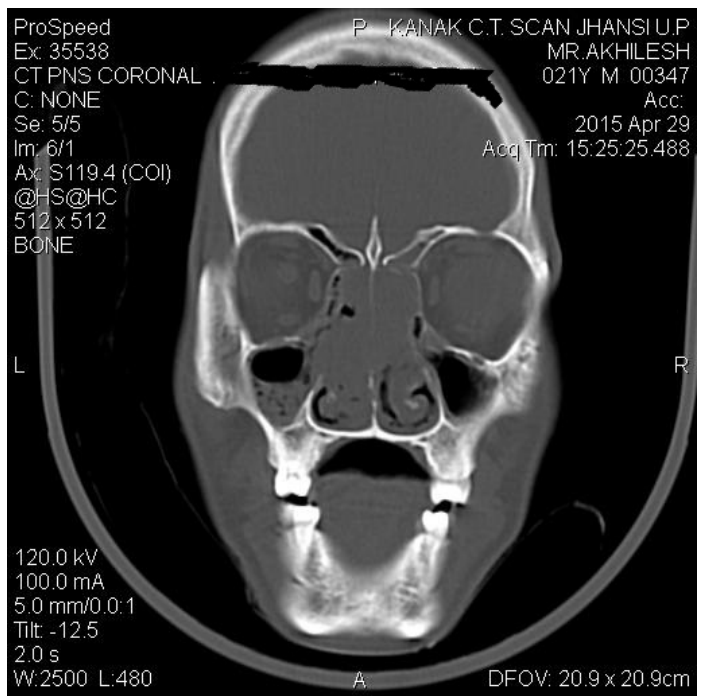

Figure 2. Case 1: CT PNS coronal view showing tumor occupying the nasal cavity with sinuses involvement.

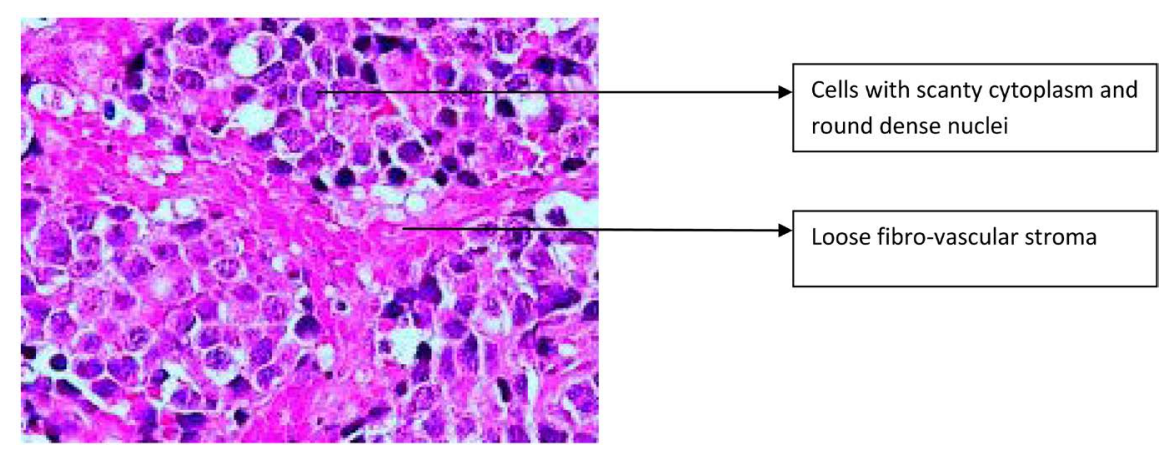

Figure 3. Case 1: Histopathological section showing cellular tissue with round dense nuclei and scanty cytoplasm. Cells are arranged in compact masses with separated by loose fibrovascular stroma. 


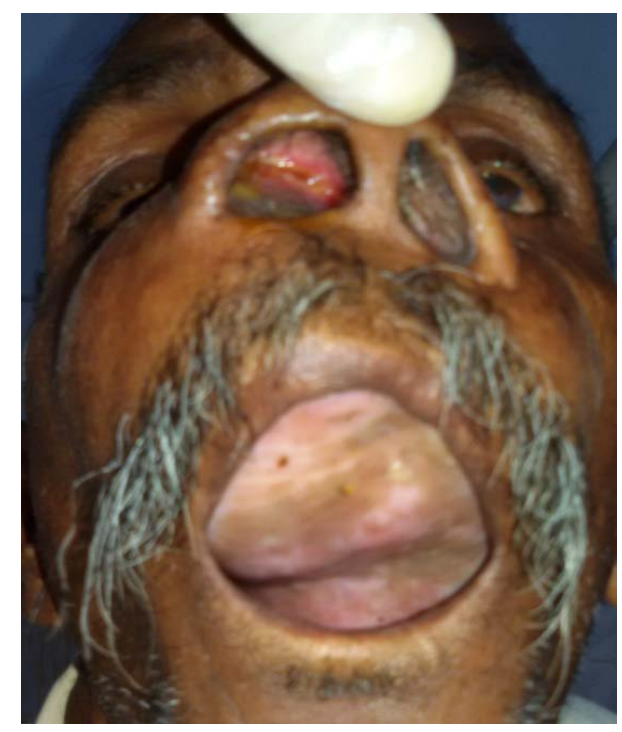

Figure 4. Case 2: Tumor occupying bilateral nasal cavity and pressing palate downwards.

Computed tomography revealed large soft tissue mass filling the entire nasal cavity. Anteriorly nasal septum was pushed towards left side; posteriorly nasal septum was not visualized. Bilateral frontal, ethmoidal and sphenoidal sinuses were opacified. Hard palate was pushed inferiorly and was thinned out. Anteriorly the mass was extending up to external nostril and posteriorly up to the nasopharynx, superiorly up to nasal roof and inferiorly up to hard palate. No obvious erosion of floor of anterior cranial fossa was seen. No intracranial extension was seen (Figure 5).

Microscopic examination of the incisional biopsy and surgical specimen showed highly vascular cellular tumor tissue consisting of proliferating uniformly small round to oval cells having large round hyperchromatic nucleus and scanty cytoplasm to form confluent masses, with occasional pseudorosettes separated by finely fibrillar stroma infiltrated by few inflammatory cells. Incisional biopsy suggested diagnosis towards round cell tumor (Figure 6).

Surgery and chemotherapy was advised to the patient but he refused any kinds of treatments.

\section{Discussion}

Sinonasal neuroendocrine malignancies are complex and rare with Esthesioneuroblastoma representing the most undifferentiated end of the spectrum of neuroendocrine tumors [7]. Esthesioneuroblastoma originates from olfactory epithelium in the upper nasal cavity in the region of the cribriform plate. Esthesioneuroblastoma accounts for approximately $3 \%$ to $6 \%$ of nasal cavity and paranasal sinus cancer cases, $0.3 \%$ of upper aero digestive tract malignancies and less than $1 \%$ of all head and neck cancers. This tumor is found equally in man and woman and occurs over a wide age range, though a bimodal age distribution with an early peak from 11 to 20 years and a later peak between 50 and 60 years of age has been reported [2]. Approximately 1300 cases have been identified since Berger and Luc described the first case in 1924 as esthesioneuroepithelioma olfactif.

Esthesioneuroblastoma is characterized by slow progression and locally aggressive behavior, which lead to long-term survival but very frequent late local recurrence. The aggressiveness of esthesioneuroblastoma is partly due to their complex anatomical location, close to vital structures, which is associated with non-specific symptoms that lead to delay in the patient diagnosis. The reported cases revealed atypical esthesioneuroblastoma identified by nasal symptoms, with radiographic images that suggested a polypoidal mass occupying the sinuses and nasal cavity and nasopharynx.

The diagnosis of esthesioneuroblastoma via light microscopy by itself can be difficult since the tumor tends to exhibit little or no differentiation. Pathological classification is challenging because the tumors must be differentiated from other round cell neoplasms of the nasal cavity such as Non-Hodgkin's lymphoma, Ewing's sarcoma, mucosal malignant melanoma and neuroendocrine carcinomas [8]. 


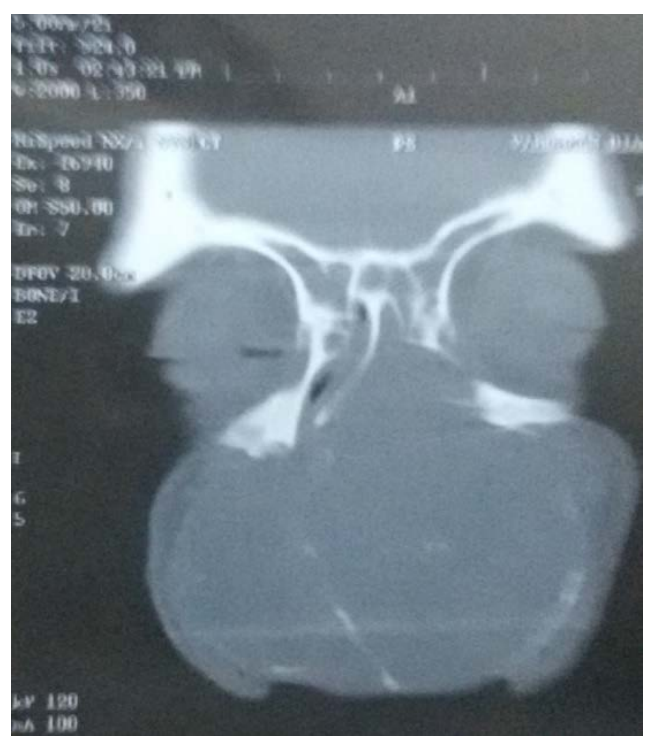

Figure 5. Case 2: CT scan showing sinuses involvement without intracranial extension.

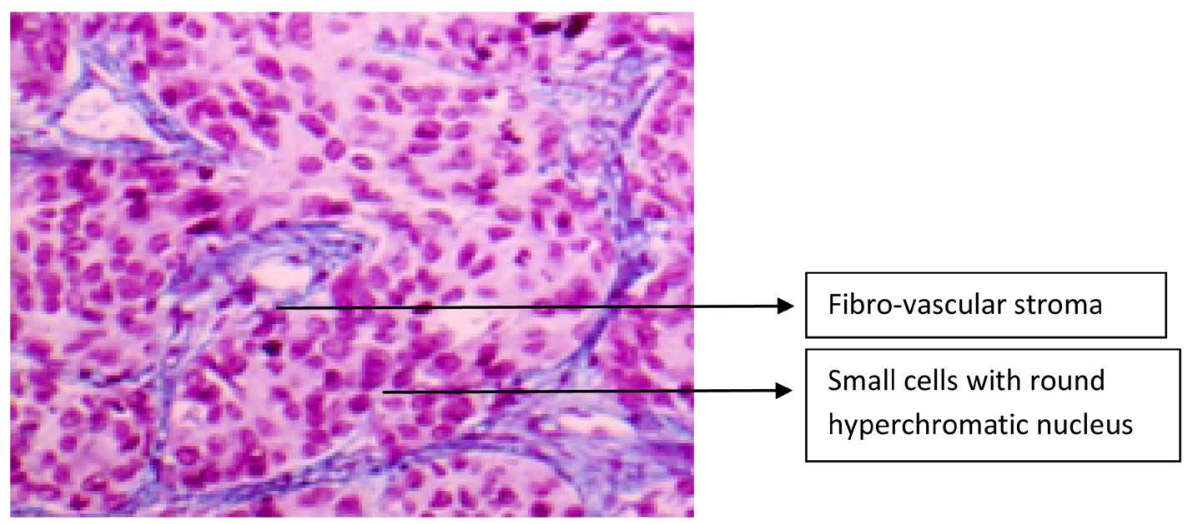

Figure 6. Case 2: Histopathological section showing highly vascular tissue consisting of proliferating small round cells having large round hyperchromatic nucleus, scanty cytoplasm, separated by finely fibrillar, stroma, infiltrated by small numbers of inflammatory cells.

The histopathological parameters that help in differentiating these tumors include the pattern of tumor cell arrangement, stroma, nuclear chromatin characteristics, presence or absence of neutrophil and rossetting. The use of a broad panel of antibodies in immunohistochemical staining may help to establish a final diagnosis. Esthesioneuroblastoma is usually positive for general neuroendocrine markers, such as neuron specific enolase (NSE), S-100 protein, synaptophysin (Syn) and chromogranin. Esthesioneuroblastoma is typically the most positive on immunohistochemical staining. ENB shows S-100 protein positive peripheral dendritic cells corresponding to Schwann cells present within the neoplasm or at the edges of tumor nests. Positivity varies in the cases reported in the literature for vimentin, keratin, glial fibrillary acidic protein, and neurofilaments [9] [10]. In the reported case the tumor showed strong positive expression of NSE, synaptophysin and vimentin. Significant correlation between CD44 expression and the stage of the disease has been suggested to help in predicting the clinical outcome. CD44s negative tumors are significantly correlated with the lack of differentiation. Thus over-expression of CD44s could be considered as a predictor of absence of infiltration of the tumor and neuroblastic tumors subtypes with favorable prognosis [11]. Staging and 5 year survival of esthesioneuroblastoma was given by Kadish in 1976 (Table 1). ENB was classified according to TNM system by DULGUEROV et CALCATERRA (1992) (Table 2).

The rates of primary tumor recurrence vary and most of the case series show local recurrence rates of approximately $14 \%$ to $30 \%$. The mean time of recurrence is 2 years, but recurrences can occur as late as 10 years after the initial diagnosis, with approximately $50 \%$ of them occurring after 5 years [9] [10] [12]. 
Table 1. Clinical classifications of esthesioneuroblastoma KADISH (1976) [15].

\begin{tabular}{ccc}
\hline Stage & Location & $\mathbf{5}$ year survival \\
\hline Stage A & Tumor localized to the nasal cavity & $75 \%$ \\
Stage B & Spread to sinuses & $68 \%$ \\
Stage C & Extension over paranasal sinuses & $41 \%$ \\
\hline
\end{tabular}

\begin{tabular}{cc} 
Table 2. TNM classification according to DULGUEROV et CALCATERRA (1992). \\
\hline T1 & Tumor localized to the nasal cavity and paranasal sinuses with a space between tumor and lamina cribosa \\
T2 & Tumor developed in nasal cavity or sinuses but in contact with cribriform lamina and/or sphenoid extension \\
T3 & Tumor with intracranial extradural and/or orbital expansion \\
T4 & Tumor with intracranial intradural extension \\
N0 & No metastatic cervical nodes \\
N1 & Metastatic cervical nodes \\
M0 & No distant metastases \\
M1 & Distant metastases \\
\hline
\end{tabular}

Craniofacial resection with definitive or adjuvant radiotherapy has been used for local control. Chemotherapy can be used in an adjuvant or neoadjuvant attempt and also in the metastatic phase or recurrent or advanced disease, although its effectiveness has still not been established. Such multimodality therapy has become the most common approach to esthesioneuroblastoma [13] [14].

According to above classification, the tumor of both the patient can be categorized as intermediate grade malignancy (T2NOM0) and along with clinical stage (Kadish B.). Endoscopic resection of tumor was done in first case and postoperatively all the symptoms of the patient resolved. Second patient refused all kinds of treatment.

\section{Conclusion}

This study highlights the characteristics and clinical features of ENB with nasal cavity and nasopharyngeal involvement and its importance in the differential diagnosis of sinonasal neuroendocrine malignancies.

\section{Affiliation}

Nil.

\section{References}

[1] Lund, V.J. and Milroy, C. (1993) Olfactory Neuroblastoma: Clinical and Pathological Aspects. Rhinology, 31, 1-6.

[2] Elkon, D. and Hightower, S.I. (1979) Esthesioneuroblastoma. Cancer, 44, 1087-1094. http://dx.doi.org/10.1002/1097-0142(197909)44:3<1087::AID-CNCR2820440343>3.0.CO;2-A

[3] Palacios \& Valvassori, 1998; Hwang, et al.; Tamase, et al., 2004; Lin, et al.; Ghaffar \& Salahuddin, 2005; Yu, et al.; Iliades, et al., 2002; Thompson, 2009.

[4] Dulguerov, P., Allal, A.S. and Calcaterra, T.C. (2001) Esthesioneuroblastoma: A Meta Analysis and Review. The Lancet Oncology, 2, 683-690. http://dx.doi.org/10.1016/S1470-2045(01)00558-7

[5] Mills, S.E. and Frierson Jr., H.F. (1985) Olfactory Neuroblastoma: A Clinicopathological Study of 21 Cases. The American Journal of Surgical Pathology, 9, 317-327. http://dx.doi.org/10.1097/00000478-198505000-00001

[6] Batsakis, J.G. (1979) Tumors of the Head and Neck. 2nd Edition, Williams and Wilkins, Baltimore, 338-349.

[7] Wenig, B.M., Prasad, M.L., Dulguerov, P., Fanburg, J.C., Kapadia, S.B. and Thomson, L.D. (2005) Neuroectodermal tumors. In: Barnes, L., Eveson, J.W., Reichart, P. and Sidransky, D., Eds., WHO Classification of Tumors, Pathology and Genetics, Head and Neck Tumors, IARC Press, Lyon, 65-75. 
[8] Thompson, L.D. (2009) Olfactory Neuroblastoma. Head and Neck Pathology, 3, 252-259. http://dx.doi.org/10.1007/s12105-009-0125-2

[9] Menon, S., Pai, P., Sengar, M., Aggarwal, J.P. and Kane, S.V. (2010) Sinonasal Malignancies with Neuroendocrine Differentiation: Case Series and Review of Literature. Indian Journal of Pathology \& Microbiology, 53, 28-34. http://dx.doi.org/10.4103/0377-4929.59179

[10] Zhang, M., Zhou, L., Wang, D.H., Huang, W.T. and Wang, S.Y. (2010) Diagnosis and Management of Esthesioneuroblastoma. ORL: Journal for Oto-Rhino-Laryngology and Its Related Specialties, 72, 113-118. http://dx.doi.org/10.1159/000278255

[11] Tabyaoui, I., Tahiri-Jouti, N., Serhier, Z., Bennani-Othmani, M., Sibai, H., Itri, M., Benchekroun, S. and Zamiati, S. (2013) Immunohistochemical Expression of CD44s in Human Neuroblastic Tumors: Moroccan Experience and Highlights on Current Data. Diagnostic Pathology, 8, 39. http://dx.doi.org/10.1186/1746-1596-8-39

[12] Gore, M.R. and Zanation, A.M. (2009) Salvage Treatment of Late Neck Metastasis in Esthesioneuroblastoma: A Meta-Analysis. Archives of Otolaryngology—Head \& Neck Surgery, 135, 1030-1034. http://dx.doi.org/10.1001/archoto.2009.143

[13] Kane, A.J., Sughrue, M.E., Rutkowski, M.J., Aranda, D., Mills, S.A., Buencamino, R., Fang, S., Barani, I.J. and Parsa, A.T. (2010) Post Treatment Prognosis of Patients with Esthesioneuroblastoma. Journal of Neurosurgery, 113, 340-351. http://dx.doi.org/10.3171/2010.2.JNS091897

[14] Platek, M.E., Merzianu, M., Mashtare, T.L., Popat, S.R., Rigual, N.R., Warren, G.W. and Singh, A.K. (2011) Improved Survival Following Surgery and Radiation Therapy for Olfactory Neuroblastoma: Analysis of the SEER Database. Radiation Oncology, 6, 41. http://dx.doi.org/10.1186/1748-717X-6-41

[15] Kadish, S., Goodman, M. and Wang, C.C. (1976) Olfactory Neuroblastoma. A Clinical Analysis of 17 Cases. Cancer, 23, 1571-1676. http://dx.doi.org/10.1002/1097-0142(197603)37:3<1571::AID-CNCR2820370347>3.0.CO;2-L 